\title{
Spin-1 bosons with coupled ground states in optical lattices
}

\author{
K. V. Krutitsky and R. Graham \\ Fachbereich Physik der Universität Duisburg-Essen, Campus Essen, Universitätsstr. 5, 45117 Essen, Germany
}

(Dated: November 12, 2018)

\begin{abstract}
The superfluid-Mott-insulator phase transition of ultracold spin-1 bosons with ferromagnetic and antiferromagnetic interactions in an optical lattice is theoretically investigated. Two counterpropagating linearly polarized laser beams with the angle $\theta$ between the polarization vectors (lin- $\theta$-lin configuration), driving an $F_{g}=1$ to $F_{e}=1$ internal atomic transition, create the optical lattice and at the same time couple atomic ground states with magnetic quantum numbers $m= \pm 1$. Due to the coupling the system can be described as a two-component one. At $\theta=0$ the system has a continuous isospin symmetry, which can be spontaneously broken, thereby fixing the number of particles in the atomic components. The phase diagram of the system and the spectrum of collective excitations, which are density waves and isospin waves, are worked out. In the case of ferromagnetic interactions, the superfluid-Mott-insulator phase transition is always second order, but in the case of antiferromagnetic interactions for some values of system parameters it is first order and the superfluid and Mott phases can coexist. Varying the angle $\theta$ one can control the populations of atomic components and continuously turn on and tune their asymmetry.
\end{abstract}

PACS numbers: 03.75.Lm,03.75.Mn,71.35.Lk

\section{INTRODUCTION}

The investigation of quantum phase transitions (QPT's) in optical lattices has become one of the major subjects in the physics of ultracold atoms, especially after a convincing experimental realization in the system of spin-polarized ${ }^{87} \mathrm{Rb}$ [1]. One of the interesting directions in this rapidly developing field is the study of QPT's in ultracold bosonic atoms with spin degrees of freedom. Systematic studies of QPT's in spin-1 bosonic systems with antiferromagnetic interactions have been performed very recently. It has been shown that there are different Mott insulating phases such as spin singlet, nematic, and dimerized ones [2, 3, 4, 5, 6], and the meanfield analysis shows that the transition between the singlet and nematic phases is weakly first order [5, 6]. Fragmented condensates with topological excitations have been studied in Ref. [2]. In the presence of a uniform magnetic field, different polar superfluids with spins aligned along or opposite to the field direction are possible [7]. QPT's of spin-2 bosons have been studied as well [8].

In the present paper, we shall investigate QPT's of spin-1 bosons with coupled ground states in an optical lattice and their collective excitations. We consider a generalization of the setup we have studied recently [9], where the population of only two degenerate ground states with magnetic quantum numbers $m= \pm 1$ was considered. The presence of atoms in all the three ground states leaves two low-lying linear combinations, which transform in the fundamental representation of an isospin $\mathrm{SU}(2)$ which determines the symmetry and the dynamics of the resulting system. Varying the laser polarization one can continuously turn on and tune the asymmetry between the components and control the populations of components in the superfluid and the Mott phases. As we shall show, a rather rich scenario of quantum phase transitions of first and/or second order becomes accessible to experiments in this way. In the present paper, we explore the phase diagram and the low-lying excitations of the various phases. In Section II we introduce the laser configuration and the Hamil- tonian which describes the system. The Bose-Hubbard model for this system is obtained in Section III, and its isospin symmetry is discussed. Section IV gives a derivation of the phase diagram in the mean-field approximation and contains our results on possible coexistence of Mott and superfluid phases and first- and second-order phase transitions. In Section V the collective excitations consisting of Bogoliubov mode and isospin waves with or without a gap are analyzed. The final section summarizes our conclusions.

\section{SYSTEM}

We consider a system of neutral polarizable spin-1 bosonic atoms of mass $M$, possessing three Zeeman-degenerate internal ground and excited electronic states characterized by the magnetic quantum number $m=0, \pm 1\left(F_{g}=F_{e}=1\right)$, in a (quasi-) one-dimensional optical lattice. The latter is assumed to be created by two counterpropagating linearly polarized laser waves of equal amplitudes and frequencies with the wave number $k_{L}$, and the angle $\theta$ (with $0 \leq \theta \leq \pi / 2$ ) between the polarization vectors (lin- $\theta$-lin configuration) [10], and with detuning $\Delta$ from the internal atomic transition. The running laser waves form left- and right- polarized standing waves with the Rabi frequencies

$$
\Omega_{ \pm}(z)=\tilde{\Omega}_{0} \cos \left(k_{L} z \pm \theta / 2\right)
$$

which couple internal ground and excited states by $V$ and $\Lambda$ transitions. In order to avoid decoherence due to spontaneous emission, $|\Delta|$ must be much larger than the spontaneous emission rate. Because of the large detuning the excited state can be adiabatically eliminated. The resulting effective Hamiltonian for the atoms in the three degenerate ground states has 
the following form $[11,12]$ :

$$
\begin{aligned}
H & =\int d z\left(\frac{\hbar^{2}}{2 M} \frac{\partial \hat{\psi}_{\alpha}^{\dagger}}{\partial z} \frac{\partial \hat{\psi}_{\alpha}}{\partial z}+\frac{\hbar}{\Delta} \hat{\psi}_{\alpha}^{\dagger} \Omega_{\alpha \beta}^{2} \hat{\psi}_{\beta}\right. \\
& \left.+\frac{g_{s}}{2} \hat{\psi}_{\alpha}^{\dagger} \hat{\psi}_{\beta}^{\dagger} \hat{\psi}_{\beta} \hat{\psi}_{\alpha}+\frac{g_{a}}{2} \hat{\psi}_{\alpha}^{\dagger} \hat{\psi}_{\alpha^{\prime}}^{\dagger} \mathbf{F}_{\alpha \beta} \cdot \mathbf{F}_{\alpha^{\prime} \beta^{\prime}} \hat{\psi}_{\beta^{\prime}} \hat{\psi}_{\beta}\right)
\end{aligned}
$$

where $\hat{\psi}_{\alpha}(z)$ is the bosonic field annihilation operator for the atom in the hyperfine ground state $|F=1, \alpha\rangle$. Summation over repeated spin indices $\alpha, \beta$ is implied. $\mathbf{F}_{\alpha \beta}$ is the vector of $F=1(3 \times 3)$ matrices in the representation furnished by the three degenerate ground states. The matrix

$$
\Omega^{2}=\left(\begin{array}{ccc}
\Omega_{+}^{2} & 0 & \Omega_{+} \Omega_{-} \\
0 & \Omega_{0}^{2} & 0 \\
\Omega_{+} \Omega_{-} & 0 & \Omega_{-}^{2}
\end{array}\right)
$$

where

$$
\Omega_{0}^{2}=\Omega_{+}^{2}+\Omega_{-}^{2}=\tilde{\Omega}_{0}^{2}\left(1+\cos \theta \cos 2 k_{L} z\right),
$$

determines the lattice potential with the period $\pi / k_{L}$ and at the same time couples the atomic ground states with $m=$ \pm 1 . The parameters $g_{s, a}$ describe the repulsive interaction of the condensate atoms and the spin-changing collisions. In a (quasi-) one-dimensional case they have the form [13] $g_{s, a}=4 \hbar^{2} a_{s, a} / M a_{\perp}^{2}$, where $a_{s, a}$ are symmetric and antisymmetric scattering lengths, and $a_{\perp}=\sqrt{2 \hbar / M \omega_{\perp}}$ is the size of the ground state for the harmonic potential with the frequency $\omega_{\perp}$ confining the atomic system in the transverse directions. In our previous work [9], we have studied the case when the ground state with $m=0$ is not occupied. In the present paper, we shall deal with the situations when this state is occupied as well.

\section{BOSE-HUBBARD MODEL}

The $V$ and $\Lambda$ laser-induced transitions lead to two sets of orthogonal Bloch eigenmodes which we denote by the indices 0 and $\Lambda$, respectively. We assume that the atoms stay always in the lowest Bloch bands with the dispersion relations $E_{0}(k)$ and $E_{\Lambda}(k)$. As explained below, in the present work we are interested in the case of $\Delta<0$ and $\theta \approx 0$. In this special case the lowest-band approximation is well justified, because the lowest Bloch bands are separated from the first excited ones by an energy of the order of $\sqrt{E_{R} \hbar \tilde{\Omega}_{0}^{2} /|\Delta|}$, where $E_{R}=$ $\hbar \omega_{R}$ is the recoil energy. Then the spinor-field operator $\hat{\boldsymbol{\Psi}}$ can be decomposed in the Wannier basis localized at the minima of the lattice potential labelled by $i$,

$$
\hat{\boldsymbol{\Psi}}(z)=\sum_{i} \sum_{\sigma=0, \Lambda} \exp \left(i \varphi_{\sigma i}\right) \mathbf{W}_{\sigma i}(z) \hat{a}_{\sigma i}
$$

where $\mathbf{W}_{\sigma i}(z)=\mathbf{W}_{\sigma}\left(z-z_{i}\right)$ are three-component Wannier spinors for the lowest energy bands. They are obtained by the solution of the eigenvalue problem for the Hamiltonian (2) in the case $g_{s, a}=0$ and satisfy the orthonormality condition $\int \mathbf{W}_{\sigma i}^{\dagger}(z) \cdot \mathbf{W}_{\sigma^{\prime} j}(z) d z=\delta_{i j} \delta_{\sigma \sigma^{\prime}}$. They have the form

$$
\mathbf{W}_{0 i}=\left(0, W_{0 i}, 0\right)^{T}, \quad \mathbf{W}_{\Lambda i}=\left(W_{+i}, 0, W_{-i}\right)^{T} .
$$

The indices $i, j$ label the sites of the one-dimensional periodic lattice. The $a_{\sigma i}$ is the Bose annihilation operator attached to the $i$ th lattice site. The phases $\varphi_{\sigma i}$ are not yet defined and their proper choice is discussed in a moment. The hyperfine spin variable at lattice site $i$ is given by

$$
\hat{\mathbf{F}}_{i}=\sum_{\sigma \sigma^{\prime}} \hat{a}_{\sigma i}^{\dagger} \mathbf{F}_{\sigma \sigma^{\prime} i} \hat{a}_{\sigma^{\prime} i} e^{-i\left(\varphi_{\sigma i}-\varphi_{\sigma^{\prime} i}\right)}
$$

with

$$
\mathbf{F}_{\sigma \sigma^{\prime} i}=\int d z \mathbf{W}_{\sigma i}^{\dagger}(z) \cdot \mathbf{F}_{i} \cdot \mathbf{W}_{\sigma^{\prime} i}(z) .
$$

Substituting Eq. (5) into Eq. (2) and taking into account only the hopping between the nearest lattice sites and the onsite atomic interactions, we obtain the two-component BoseHubbard Hamiltonian

$$
\begin{aligned}
& \hat{H}_{B H}=-\sum_{\sigma}\left|J_{\sigma}\right| \sum_{<i, j>} \hat{a}_{\sigma i}^{\dagger} \hat{a}_{\sigma j} \\
& +\sum_{\sigma} \frac{U_{\sigma}}{2} \sum_{i} \hat{n}_{\sigma i}\left(\hat{n}_{\sigma i}-1\right)+K \sum_{i} \hat{n}_{0 i} \hat{n}_{\Lambda i} \\
& -\frac{|P|}{2} \sum_{i}\left(\hat{a}_{0 i}^{\dagger} \hat{a}_{0 i}^{\dagger} \hat{a}_{\Lambda i} \hat{a}_{\Lambda i}+\hat{a}_{\Lambda i}^{\dagger} \hat{a}_{\Lambda i}^{\dagger} \hat{a}_{0 i} \hat{a}_{0 i}\right) \\
& -\delta \sum_{i} \hat{n}_{0 i}-\mu \sum_{\sigma, i} \hat{n}_{\sigma i},
\end{aligned}
$$

where $\mu$ is a chemical potential. The tunneling matrix elements

$$
J_{\sigma}=-\int \mathbf{W}_{\sigma, i+1}^{\dagger}(z) \cdot\left(-\frac{\hbar^{2}}{2 M} \frac{\partial^{2}}{\partial z^{2}}+\frac{\hbar}{\Delta} \hat{\Omega}^{2}\right) \cdot \mathbf{W}_{\sigma, i}(z) d z
$$

the atomic interaction parameters

$$
\begin{aligned}
U_{\Lambda}= & \int\left[\left(g_{s}+g_{a}\right)\left(\left|W_{+i}\right|^{2}+\left|W_{-i}\right|^{2}\right)^{2}\right. \\
& \left.-4 g_{a}\left|W_{+i}\right|^{2}\left|W_{-i}\right|^{2}\right] d z \\
U_{0}= & g_{s} \int\left|W_{0 i}\right|^{4} d z \\
K= & \left(g_{s}+g_{a}\right) \int\left|W_{0 i}\right|^{2}\left(\left|W_{+i}\right|^{2}+\left|W_{-i}\right|^{2}\right) d z \\
P= & 2 g_{a} \int\left(W_{0 i}^{*}\right)^{2} W_{+i} W_{-i} d z
\end{aligned}
$$

and the relative shift of the mean energies of the eigenmodes

$$
\begin{aligned}
\delta & =\int \mathbf{W}_{\Lambda, i}^{\dagger}(z) \cdot\left(-\frac{\hbar^{2}}{2 M} \frac{\partial^{2}}{\partial z^{2}}+\frac{\hbar}{\Delta} \hat{\Omega}^{2}\right) \cdot \mathbf{W}_{\Lambda, i}(z) d z \\
& -\int \mathbf{W}_{0, i}^{\dagger}(z) \cdot\left(-\frac{\hbar^{2}}{2 M} \frac{\partial^{2}}{\partial z^{2}}+\frac{\hbar}{\Delta} \hat{\Omega}^{2}\right) \cdot \mathbf{W}_{0, i}(z) d z \\
& =\frac{1}{k_{L}} \int_{0}^{k_{L}}\left[E_{\Lambda}(k)-E_{0}(k)\right] d k
\end{aligned}
$$


can be simultaneously changed by varying the laser intensity (which is proportional to $\tilde{\Omega}_{0}^{2}$ ) and/or the angle $\theta$, but the variations of $J_{\sigma}$ and $\delta$ are much faster. The parameter $P$ can be either positive or negative depending on the sign of the antisymmetric coupling $g_{a}$.

The phases $\varphi_{\sigma i}$ are determined from the requirement of minimal energy of the Bose-Hubbard Hamiltonian, which amounts to demanding that $J_{\sigma} \cos \left(\varphi_{\sigma i}-\varphi_{\sigma, i+1}\right)$ and $P \cos \left(2 \varphi_{0 i}-2 \varphi_{\Lambda i}\right)$ are maximal. This requirement leading to

$$
\begin{array}{r}
\varphi_{\sigma, i+1}= \begin{cases}\varphi_{\sigma i} & \text { if } J_{\sigma}>0 \\
\varphi_{\sigma i} \pm \pi & \text { if } J_{\sigma}<0\end{cases} \\
\varphi_{0 i}= \begin{cases}\varphi_{\Lambda i} & \text { if } g_{a}<0 \\
\varphi_{\Lambda i} \pm \pi / 2 & \text { if } g_{a}>0\end{cases}
\end{array}
$$

has been taken into account in the derivation of the Hamiltonian (9), which therefore only depends on the absolute values $\left|J_{\sigma}\right|$ and $|P|$ besides the other system parameters $U_{\sigma}$, which are positve in the examples we consider and of about equal size, $K$, which can be larger or smaller than the $U_{\sigma}$ depending on the sign of the antisymmetric coupling $g_{a}$, and $\delta$. As one can clearly see from Eq. (4) $J_{0}$ depends on $\Omega_{0}^{2} \cos \theta /|\Delta|$ and it is always positive [14]. In principle $J_{\Lambda}$ can also become negative for large enough $\theta$ [9], but at such values of $\theta$ the shift $\delta$ becomes comparable to the atomic interaction parameters in Hamiltonian (9) and it becomes energetically more favorable for atoms to stay in the 0 mode. In the present work, we are interested in the situations when both modes are occupied. Therefore we have to restrict ourselves to small values of $\theta$ and consider $J_{\Lambda}$ as well as $J_{0}$ as positive quantities. Thus in the following always the first of the alternatives in Eq. (13) applies. In general, $J_{0} \geq J_{\Lambda}$.

In the present paper, we investigate only the case of red detuning $(\Delta<0)$, since in this case the minima of the effective periodic potentials for the $\Lambda$ and 0 modes coincide which leads to higher values of $K$ and $|P|$ compared to the case of blue detuning $(\Delta>0)$. In addition if $\theta=0$, $W_{+i}=W_{-i}=W_{0 i} / \sqrt{2}$ and we have

$$
J_{0}=J_{\Lambda}=J, U_{0}=U_{\Lambda}=U, K=U+P, \delta=0 .
$$

The 0 and $\Lambda$ modes are degenerate in this case, because $E_{0}(k)=E_{\Lambda}(k)$ for any value of the laser intensity. Indeed the Hamiltonian (9) then has an enhanced $\mathrm{U}(1) \times \mathrm{U}(1)$ symmetry. This can be seen by defining the global generators of an SU(2) algebra (with $L$ the number of lattice sites)

$$
\begin{aligned}
& \hat{T}_{1}=\frac{1}{2 L} \sum_{i=1}^{L}\left(\hat{a}_{\Lambda i}^{\dagger} \hat{a}_{0 i}+\hat{a}_{0 i}^{\dagger} \hat{a}_{\Lambda i}\right), \\
& \hat{T}_{2}=\frac{i}{2 L} \sum_{i=1}^{L}\left(\hat{a}_{\Lambda i}^{\dagger} \hat{a}_{0 i}-\hat{a}_{0 i}^{\dagger} \hat{a}_{\Lambda i}\right), \\
& \hat{T}_{3}=\frac{1}{2 L} \sum_{i=1}^{L}\left(\hat{a}_{0 i}^{\dagger} \hat{a}_{0 i}-\hat{a}_{\Lambda i}^{\dagger} \hat{a}_{\Lambda i}\right) .
\end{aligned}
$$

In terms of these generators the density of the hyperfine spin variable $\hat{\mathbf{f}}=\sum_{i} \hat{\mathbf{F}}_{i} / L$ for $\theta=0$, which always points in the
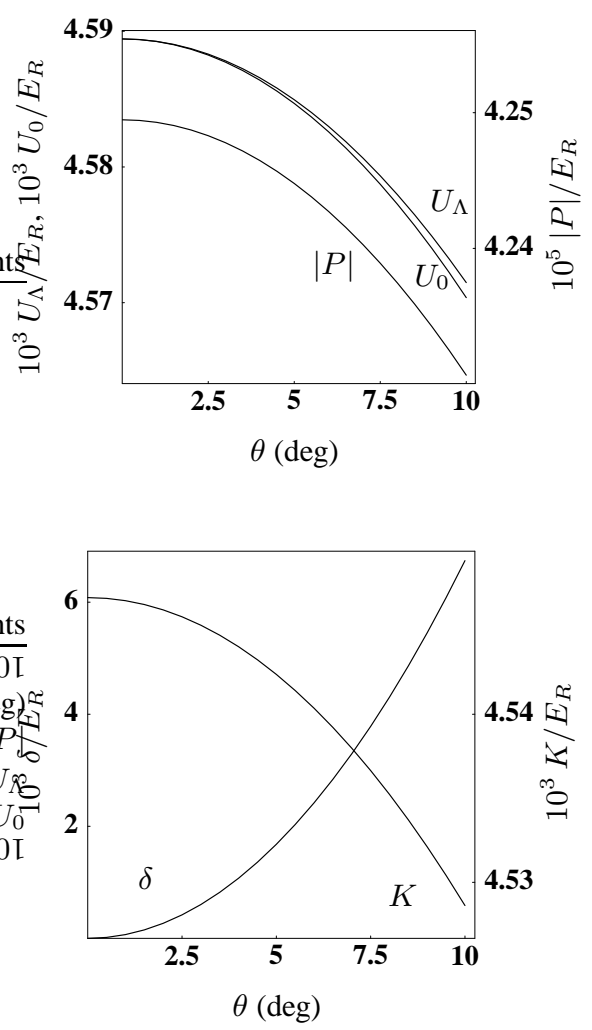

FIG. 1: Dependences of the parameters in the Bose-Hubbard Hamiltonian (9) on $\theta$ for ${ }^{87} \mathrm{Rb}, q=\tilde{\Omega}_{0}^{2} / 4 \omega_{R} \Delta=-5$.

$x$ direction defined by the direction of the linear polarization of the external laser, is given by $\hat{\mathbf{f}}=2 \hat{T}_{1(2)} \mathbf{e}_{x} / L$ for $g_{a}<0$ $\left(g_{a}>0\right)$.

Together with the number density $\hat{n}=\sum_{i=1}^{L}\left(\hat{a}_{0 i}^{\dagger} \hat{a}_{0 i}+\right.$ $\left.\hat{a}_{\Lambda i}^{\dagger} \hat{a}_{\Lambda i}\right) / L$, the $\hat{\mathbf{T}}$ generates an U(2) algebra. However, for $g_{a}>0\left(g_{a}<0\right)$ the Hamiltonian (9) only commutes with the generators $\hat{n}$ and $\hat{T}_{1}\left(\hat{T}_{2}\right)$ of the respective $\mathrm{U}(1) \times \mathrm{U}(1)$ subgroups which they generate.

Increasing the angle $\theta$, one changes the form of dispersions $E_{\sigma}(k)$ and Wannier spinors $\mathbf{W}_{\sigma i}(z)$, and these changes are different for the $\Lambda$ and 0 modes. As a consequence, the values of the parameters in Hamiltonian (9) will be modified (see Ref. [14] for $J_{0}\left[\left(\tilde{\Omega}_{0}^{2} / \Delta\right) \cos \theta\right]$, Ref. [9] for $J_{\Lambda}(\theta)$, and Fig. 1 for the others) and relations (15) become violated. Therefore, varying the angle $\theta$ one can continuously tune the parameters in the Hamiltonian (9) and remove the degeneracy by reducing the symmetry to the $\mathrm{U}(1)$ generated by $\hat{n}$.

Throughout the paper in our numerical estimations we have used known values of scattering lengths of ${ }^{87} \mathrm{Rb}\left(g_{a}<0\right)$ and ${ }^{23} \mathrm{Na}\left(g_{a}>0\right)[11]$ and $\omega_{\perp}=2 \pi \times 200 \mathrm{~Hz}$. The laser wavelengths $\lambda_{L}$ were chosen to be $780 \mathrm{~nm}$ and $600 \mathrm{~nm}$, respectively, which corresponds to the D1 line.

Despite the fact that Hamiltonian (9) has been explicitly derived here only for a one-dimensional lattice, it can be realized in higher-dimensional optical lattices as well. This can be 
done with the aid of additional pairs of linearly polarized laser beams propagating along the $x$ and $y$ axes. In order to avoid the interference of laser beams propagating in the orthogonal directions their frequencies must be sufficiently different. In order not to change the number of components in the BoseHubbard Hamiltonian, the scheme of the internal atomic transitions should remain the same. This can be achived if there is no $\pi$ component of the laser field, i.e., all the laser beams must be polarized in a plane perpendicular to the quantization axis, which is chosen to be the $z$ axis. Therefore the polarization vectors of the laser beams propagating along the $y$ and $x$ axes are to be parallel to the $x$ and $y$ axes, respectively. As discussed above and confirmed by numerical calculations in the next section, the angle $\theta$ must be very small and then $J_{\Lambda} \approx J_{0}$. Thus it is not difficult to adjust the laser intensities and detunings of the additional laser beams such that the anisotropy of the tunneling matrix elements in the higher-dimensional version of the Hamiltonian 9) will be negligible.

\section{MEAN-FIELD APPROXIMATION AND THE GROUND-STATE ENERGY}

In order to investigate the superfluid-Mott-insulator phase transition, we employ the mean-field approximation [15, 16]

$$
\hat{a}_{\sigma i}^{\dagger} \hat{a}_{\sigma j} \approx \psi_{\sigma}\left(\hat{a}_{\sigma j}+\hat{a}_{\sigma i}^{\dagger}\right)-\psi_{\sigma}^{2}
$$

where $\psi_{\sigma}$ is the order parameter for Bose-Einstein condensation in the component $\sigma$. The $\psi_{\sigma}$ carry phases, which are in equilibrium either the same for the both components or differ by $\pi$, as can be easily shown by energy minimization. Thus we may take the $\psi_{\sigma}$ as real without unwarranted loss of generality. In this approximation, the Bose-Hubbard Hamiltonian becomes local and every lattice site is described by the Hamiltonian

$$
\begin{aligned}
& \hat{H}_{B H}^{\prime}=-2 d \sum_{\sigma} J_{\sigma}\left[\left(\hat{a}_{\sigma}^{\dagger}+\hat{a}_{\sigma}\right) \psi_{\sigma}-\psi_{\sigma}^{2}\right] \\
& +\sum_{\sigma} \frac{U_{\sigma}}{2} \hat{n}_{\sigma}\left(\hat{n}_{\sigma}-1\right)+K \hat{n}_{0} \hat{n}_{\Lambda} \\
& -\frac{|P|}{2}\left(\hat{a}_{0}^{\dagger} \hat{a}_{0}^{\dagger} \hat{a}_{\Lambda} \hat{a}_{\Lambda}+\hat{a}_{\Lambda}^{\dagger} \hat{a}_{\Lambda}^{\dagger} \hat{a}_{0} \hat{a}_{0}\right)-\delta \hat{n}_{0}-\mu \sum_{\sigma} \hat{n}_{\sigma},
\end{aligned}
$$

where $d$ is the dimensionality of the lattice and we have omitted the site index $i$. Since in the mean-field approximation $d$ plays a role of a numerical parameter, we have done the calculations presented below for $d=1$. In the case of $d=2,3$, there will be only numerical corrections, but all the qualitative features will remain the same.

The values of the order parameters minimizing the groundstate energy $E_{g}\left(\psi_{0}, \psi_{\Lambda}\right)$ of the Hamiltonian (18) determine the phase diagram of the system at zero temperature: $\psi_{\sigma}=$ $0\left(\psi_{\sigma} \neq 0\right)$ corresponds to the non-condensed (superfluid) phase of the $\sigma$-component.

The ground-state energy $E_{g}\left(\psi_{0}, \psi_{\Lambda}\right)$ has been calculated by numerical diagonalization of the Hamiltonian 18 using
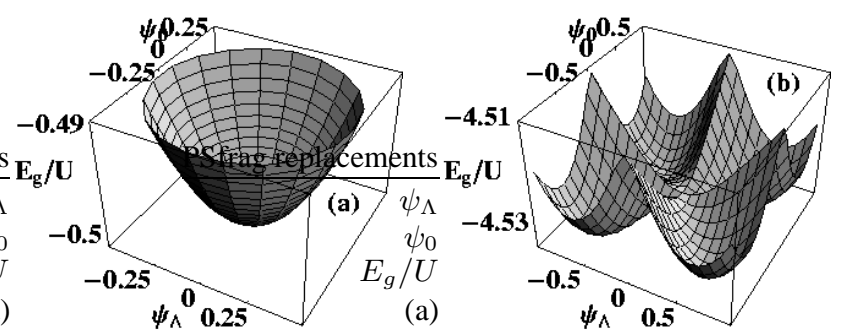

FIG. 2: Ground-state energy of the Hamiltonian 18 for ${ }^{87} \mathrm{Rb}\left(g_{a}<\right.$ 0 ) in the case $\theta=0 . q=\tilde{\Omega}_{0}^{2} / 4 \omega_{R} \Delta=-4.5, \mu / U=0.5$ (a), $\mu / U=2.5$ (b).

superpositions of the number states

$$
|s\rangle=\sum_{n_{0}=0}^{N} \sum_{n_{\Lambda}=0}^{N} c_{n_{0} n_{\Lambda}}\left|n_{0}\right\rangle\left|n_{\Lambda}\right\rangle .
$$

We have done calculations with $N=10$ and the results are discussed below.

\section{A. Ferromagnetic interactions $\left(g_{a}<0\right)$}

Typical dependences of the ground-state energy of the Hamiltonian (18) on the order parameters $\psi_{0}, \psi_{\Lambda}$ for $g_{a}<0$ are shown in Figs. 2 and 3 If $\theta=0$, the ground-state energy $E_{g}$ is independent of the sign of the real $\psi_{\sigma}$,

$$
E_{g}\left(\psi_{\Lambda}, \psi_{0}\right)=E_{g}\left(\left|\psi_{\Lambda}\right|,\left|\psi_{0}\right|\right)
$$

and it is a symmetric function of $\psi_{0}$ and $\psi_{\Lambda}: E_{g}\left(\psi_{0}, \psi_{\Lambda}\right)=$ $E_{g}\left(\psi_{\Lambda}, \psi_{0}\right)$. More generally, for complex $\psi_{\sigma}$ the isospin symmetry at $\theta=0$ demands (still for $g_{a}<0$ ) that $E_{g}$ only depends on the invariants $\left|\psi_{0}\right|^{2}+\left|\psi_{\Lambda}\right|^{2}$ and $\left(\psi_{\Lambda}^{*} \psi_{0}+\psi_{0}^{*} \psi_{\Lambda}\right)$. One has to distinguish the following two cases. (i) There is a single minimum at $\psi_{0}=\psi_{\Lambda}=0$ [Mott phase, Fig. 2] a), corresponding to a state of unbroken $\mathrm{U}(1) \times \mathrm{U}(1)$ symmetry]. (ii) There are four equal minima at $\psi_{0}^{2}=\psi_{\Lambda}^{2} \neq 0$ [symmetric two-component Bose-Einstein condensate (BEC), Fig. 2]b)]. In any of these minima the system has a nonvanishing $x$ component of the expectation value of the hyperfine spin density $\left\langle\mathbf{f}_{x}\right\rangle \sim\left\langle T_{1}\right\rangle \sim\left(\psi_{\Lambda}^{*} \psi_{0}+\psi_{0}^{*} \psi_{\Lambda}\right) / L$

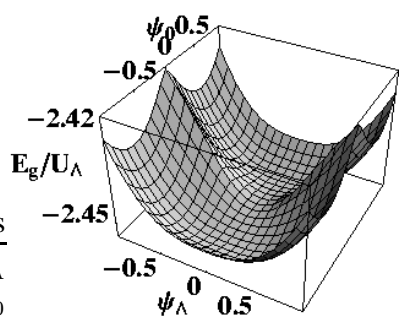

FIG. 3: Ground-state energy of the Hamiltonian 18 for ${ }^{87} \mathrm{Rb}\left(g_{a}<\right.$ $0)$ in the case $\theta=1^{\circ}, q=\widetilde{\Omega}_{0}^{2} / 4 \omega_{R} \Delta=-4.5, \mu / U_{\Lambda}=1.71$. 

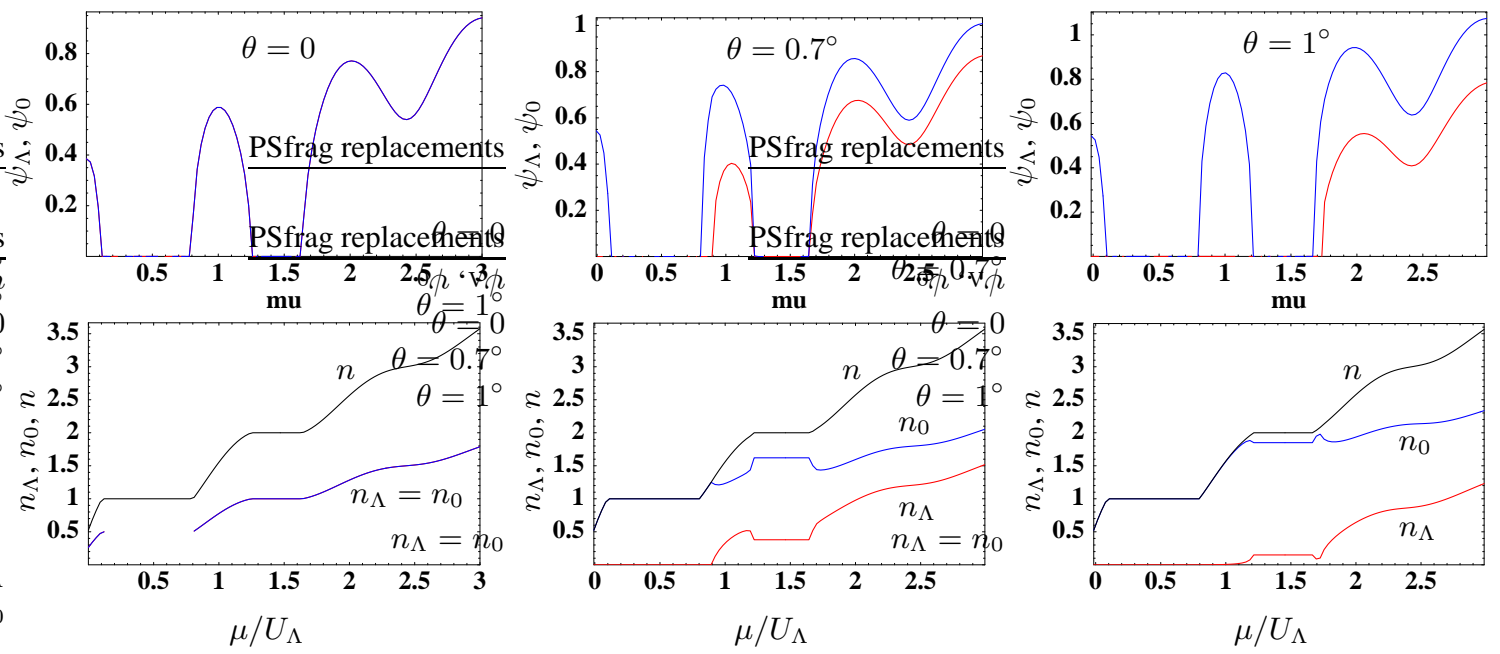

FIG. 4: Extremal values of the order parameters $\psi_{\Lambda}, \psi_{0}$ (upper row) and the mean occupation numbers of $\Lambda$ and 0 components (lower row) for ${ }^{87} \mathrm{Rb}\left(g_{a}<0\right) . q=-4.5, \theta=0$ (a), $0.7^{\circ}(\mathrm{b}), 1^{\circ}$ (c)

If $\theta \neq 0$ the scales of $\psi_{0}$ and $\psi_{\Lambda}$ become different. $E_{g}$ is not a symmetric function of $\psi_{0}$ and $\psi_{\Lambda}$ anymore, but Eq. (20) remains valid. The case (i) is possible again. Due to the different scalings of $\psi_{0}$ and $\psi_{\Lambda}$ instead of (ii) we have $\psi_{0}^{2} \neq \psi_{\Lambda}^{2} \neq 0$ (two-component BEC). In addition, if $\theta \neq 0$, there are additional cases with two equal minima of $E_{g}$ reached at (iii) $\psi_{\Lambda}=0, \psi_{0} \neq 0$ [mixture of noncondensed component $\Lambda$ and BEC of component 0, Fig. 3]. As we see in Fig. [4 the mean occupation numbers of the $\Lambda$ component turn out to be so small in these regions that there seems to be no hope to observe the noncondensed component experimentally.

The phase diagram in the case $\theta=0$ is shown in Fig. 5 The lobes correspond to the Mott phases with different particle numbers per lattice site, and outside the lobes we have a superfluid phase. The superfluid-insulator transition is always second order for $g_{a}<0$.

\section{B. Antiferromagnetic interactions $\left(g_{a}>0\right)$}

In the case of positive $g_{a}$, i.e., positive $P$, the situation is quite different. If $\theta=0$, the only condition in the ground state is $\psi_{0}^{*} \psi_{\Lambda}-\psi_{\Lambda}^{*} \psi_{0}=0$, which restricts the expectation value of the isospin $\langle\mathbf{T}\rangle$ to the $(1,3)$ plane. Apart from this restriction $E_{g}$ is a rotationally symmetric function of the order parameters for any values of $\mu$ and the dependences de-

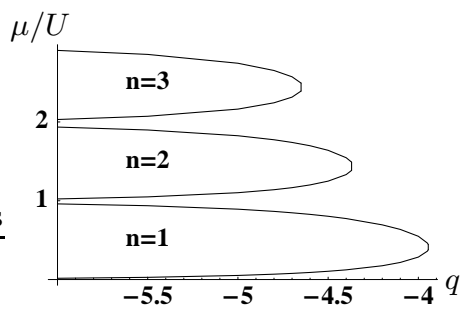

FIG. 5: Phase diagram for ${ }^{87} \mathrm{Rb}\left(g_{a}<0\right)$ in the case $\theta=0$. picted in Fig. 22 b) do not occur. Case (i) of the previous subsection remains possible, but instead of (ii) the minima of $E_{g}$ are located on a circle $\psi_{0}^{2}+\psi_{\Lambda}^{2}=\psi^{2}$ on which a point is selected by spontaneous symmetry breaking [stable two-component Bose-Einstein condensed phase with spontaneously broken isospin symmetry with $\langle\mathbf{T}\rangle$ oriented arbitrarily in the $(1,3)$ plane, Fig. [c)]. In addition, there can be not only global minima, but also local ones either at $\psi_{\Lambda}=\psi_{0}=0$ [metastable Mott phase, Fig. 6b)] or on a circle [metastable superfluid phase, Fig. [a)], depending on the system parameters. This means that in some parameter regions the superfluid and Mott phases can coexist and the phase transition can be either first or second order in contrast to the case of ferromag-
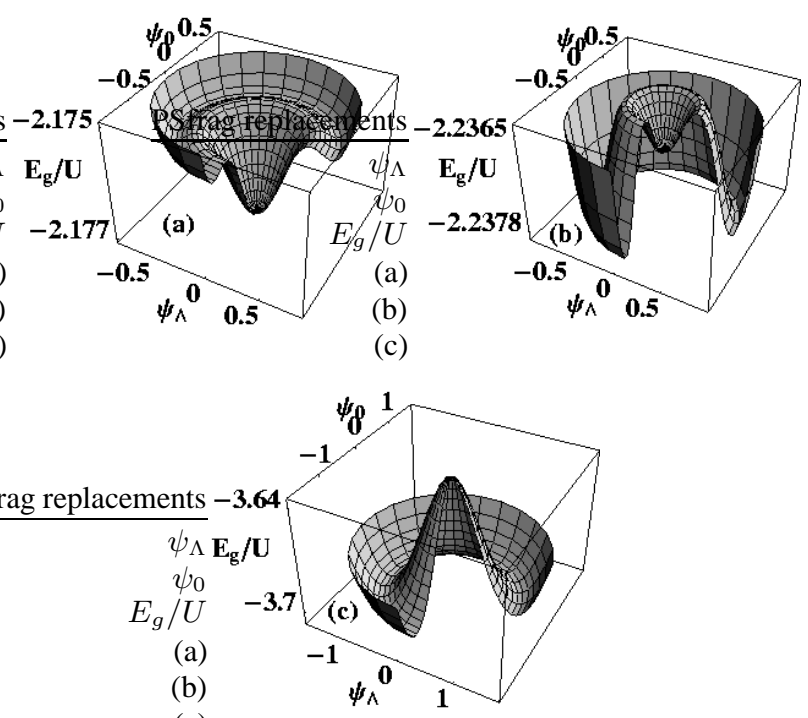

FIG. 6: Ground-state energy of the Hamiltonian (18) for ${ }^{23} \mathrm{Na}\left(g_{a}>\right.$ 0 ) in the case $\theta=0 . q=\Omega_{0}^{2} / 4 \omega_{R} \Delta=-6.1, \mu / U=1.57$ (a), $\mu / U=1.6$ (b), $\mu / U=2.2$ (c). 


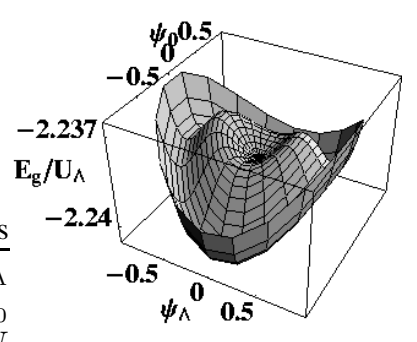

FIG. 7: Ground-state energy of the Hamiltonian 18 for ${ }^{23} \mathrm{Na}\left(g_{a}>\right.$ $0)$ in the case $\theta=0.1^{\circ} . q=\Omega_{0}^{2} / 4 \omega_{R} \Delta=-6.1, \mu / U_{\Lambda}=1.6$.

netic interactions, where it is always second order.

If $\theta \neq 0$, the symmetry between the two components is destroyed and the scalings for $\psi_{0}$ and $\psi_{\Lambda}$ become different. The minima of $E_{g}$ can be reached at either $\psi_{0}=\psi_{\Lambda}=0$ or $\psi_{\Lambda}=0, \psi_{0} \neq 0$ as in the case $g_{a}<0$ discussed above. In addition, there are dependences shown in Fig. 7 with (or without) one local minimum at $\psi_{\Lambda}=\psi_{0}=0$ and two global minima at $\psi_{\Lambda}=0, \psi_{0} \neq 0$. In contrast to the case of negative $g_{a}$, the populations of the components can be comparable in all the cases (Fig. 8).

The phase diagram in the case $\theta=0$ is shown in Fig. 9 It consists of a series of lobes corresponding to the Mott phase as in the case of ferromagnetic interactions. However, the superfluid-insulator transition for $n=2$ is now first order, while for $n=1,3$ it remains to be second order. In the case $n=2$, the stable (metastable) superfluid phase coexists with metastable (stable) Mott phase in a small region near the phase boundary.
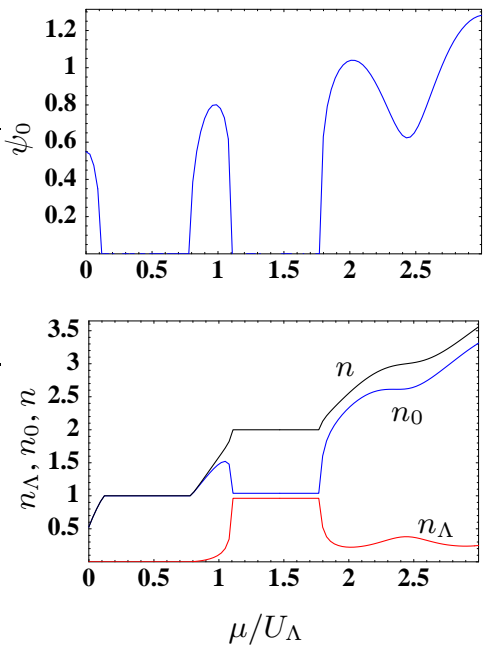

FIG. 8: Extremal values of the order parameter $\psi_{0}$ and the mean occupation numbers of $\Lambda$ and 0 components for ${ }^{23} \mathrm{Na}\left(g_{a}>0\right)$. $q=-6.5, \theta=0.1^{\circ}$.

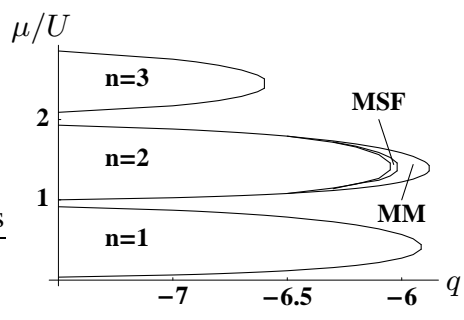

FIG. 9: Phase diagram for ${ }^{23} \mathrm{Na}\left(g_{a}>0\right)$ in the case $\theta=0$. The regions of metastable superfluid phase coexisting with the stable Mott phase and that of matastable Mott phase coexisting with the stable superfluid phase are denoted by MSF and MM, respectively.

\section{Mott phase, arbitrary $g_{a}$}

In the Mott phase, $\psi_{\Lambda}=\psi_{0}=0$ and for every number of atoms per site $n$ there are $n+1$ eigenstates of the local Hamiltonian (18). For $n \leq 3$ the expressions for eigenstates are very simple. If the number of particles per site $n$ is less than two, the terms which contain $|P|$ in Eq. (18) do not give any contribution and we have the usual Fock states,

$$
\begin{aligned}
E_{0} & =0, \quad|n=0\rangle=|0\rangle_{\Lambda}|0\rangle_{0}, \\
E_{1,0} & =-(\mu+\delta), \quad|n=1\rangle_{0}=|0\rangle_{\Lambda}|1\rangle_{0}, \\
E_{1,1} & =-\mu, \quad|n=1\rangle_{1}=|1\rangle_{\Lambda}|0\rangle_{0} .
\end{aligned}
$$

The lowest of these energies gives the ground-state energy per lattice site. The states with higher energies correspond to excited states whose positive excitation energies are the difference of the respective energies $E$ and that of the ground state. In the case $\theta=0, \delta$ vanishes. The states $|n=1\rangle_{0}$ and $|n=1\rangle_{1}$ become degenerate and any linear combination of these states forms the SU(2)-symmetric manifold of degenerate ground states in the case of one atom per lattice site. This case requires a positive chemical potential $\mu \geq 0$, and, as we shall see by comparing with the energy levels for the case of two atoms per lattice site, $\mu \leq U-|P|$. Thus, in the Mott phase with one atom per site, the mean numbers of atoms in $\Lambda$ and 0 modes $n_{\Lambda}$ and $n_{0}$ are undefined, but the total number of atoms $n=n_{\Lambda}+n_{0}$ is fixed [see Fig. 4la)]. If $\theta \neq 0$, the degeneracy is removed and $n_{\Lambda}=0, n_{0}=1$ [Figs. 4(b) and (c)].

If $n \geq 2$, the eigenstates are in general superpositions of Fock states. For $n=2$ we get

$$
\begin{aligned}
& E_{2,1}=K-\delta-2 \mu, \quad|n=2\rangle_{1}=|1\rangle_{\Lambda}|1\rangle_{0} \\
& E_{2, \pm}=\frac{U_{0}+U_{\Lambda}}{2}-\delta \pm \sqrt{\left(\frac{U_{0}-U_{\Lambda}}{2}-\delta\right)^{2}+P^{2}}-2 \mu \\
& |n=2\rangle_{ \pm}=c_{0 \pm}^{(2)}|0\rangle_{\Lambda}|2\rangle_{0}+c_{2 \pm}^{(2)}|2\rangle_{\Lambda}|0\rangle_{0} \\
& c_{0, \pm}^{(2)}=\left\{\frac{1}{2}\left[1 \pm \frac{U_{0}-U_{\Lambda}-2 \delta}{\sqrt{\left(U_{0}-U_{\Lambda}-2 \delta\right)^{2}+4 P^{2}}}\right]\right\}^{1 / 2}, \\
& c_{2, \pm}^{(2)}=\mp\left\{\frac{1}{2}\left[1 \mp \frac{U_{0}-U_{\Lambda}-2 \delta}{\sqrt{\left(U_{0}-U_{\Lambda}-2 \delta\right)^{2}+4 P^{2}}}\right]\right\}^{1 / 2} .
\end{aligned}
$$


In the case $\theta=0, c_{0, \pm}^{(2)}=1 / \sqrt{2}$ and $c_{2, \pm}^{(2)}=\mp 1 / \sqrt{2}$. For $\mu \geq U-|P|$ the ground state is either $|n=2\rangle_{-}$or an arbitrary linear combination of $|n=2\rangle_{1}$ and $|n=2\rangle_{-}$: If $g_{a}>0, \mid n=$ $2\rangle_{-}$is a ground state and the mean particle numbers $n_{\Lambda}=$ $n_{0}=1$. If $g_{a}<0, E_{2,-}=E_{2,1}=U-|P|-2 \mu<E_{2,+}$, i.e., the ground state is doubly degenerate. Nevertheless, we have again $n_{\Lambda}=n_{0}=1$.

For $n=3$

$$
\begin{aligned}
& E_{3 g \pm}=\frac{3 U_{0}+U_{\Lambda}}{2}+K-2 \delta \\
& \pm \sqrt{\left(\frac{3 U_{0}-U_{\Lambda}}{2}-K-\delta\right)^{2}+3 P^{2}-3 \mu} \\
& |n=3\rangle_{g \pm}=c_{0 \pm}^{(3)}|0\rangle_{\Lambda}|3\rangle_{0}+c_{2 \pm}^{(3)}|2\rangle_{\Lambda}|1\rangle_{0}, \\
& c_{0, \pm}^{(3)}=\left\{\frac{1}{2}\left[1 \pm \frac{3 U_{0}-U_{\Lambda}-2 K-2 \delta}{\sqrt{\left(3 U_{0}-U_{\Lambda}-2 K-2 \delta\right)^{2}+12 P^{2}}}\right]\right\}^{1 / 2} \\
& c_{2, \pm}^{(3)}=\mp\left\{\frac{1}{2}\left[1 \mp \frac{3 U_{0}-U_{\Lambda}-2 K-2 \delta}{\sqrt{\left(3 U_{0}-U_{\Lambda}-2 K-2 \delta\right)^{2}+12 P^{2}}}\right]\right\}^{1 / 2} \\
& E_{3 u \pm}=\frac{U_{0}+3 U_{\Lambda}}{2}+K-\delta \\
& |n=3\rangle_{u \pm}=c_{1 \pm}^{(3)}|1\rangle_{\Lambda}|2\rangle_{0}+c_{3 \pm}^{(3)}|3\rangle_{\Lambda}|0\rangle_{0}, \\
& c_{1, \pm}^{(3)}=\left\{\frac{1}{2}\left[1 \pm \frac{U_{0}-3 U_{\Lambda}}{\sqrt{\left(U_{0}-3 U_{\Lambda}+2 K-2 \delta\right)^{2}+12 P^{2}}}\right]\right. \\
& c_{3, \pm}^{(3)}=\mp\left\{\frac{1}{2}\left[1 \mp \frac{U_{0}-3 U_{\Lambda}+2 K-2 \delta}{\sqrt{\left(U_{0}-3 U_{\Lambda}+2 K-2 \delta\right)^{2}+12 P^{2}}}\right]\right\}^{1 / 2}
\end{aligned}
$$

$1 / 2$ with the local classical mean-field Hamiltonian

$$
\begin{aligned}
& H=-2\left(J_{0}\left|\alpha_{0}\right|^{2}+J_{\Lambda}\left|\alpha_{\Lambda}\right|^{2}\right)+\frac{U_{0}}{2}\left|\alpha_{0}\right|^{4}+\frac{U_{\Lambda}}{2}\left|\alpha_{\Lambda}\right|^{4} \\
& +K\left|\alpha_{0} \alpha_{\Lambda}\right|^{2}-\frac{|P|}{2}\left[\left(\alpha_{\Lambda}^{*} \alpha_{0}\right)^{2}+\left(\alpha_{0}^{*} \alpha_{\Lambda}\right)^{2}\right] \\
& -(\delta+\mu)\left|\alpha_{0}\right|^{2}-\mu\left|\alpha_{\Lambda}\right|^{2} .
\end{aligned}
$$

At least roughly, the $\alpha_{\sigma}$ correspond to the previously introduced order parameters $\psi_{\sigma}$, but it should be noted that the present mean-field approximation is different from the one of the previous section [Eq.[17], where it was employed only to approximate the hopping term of the Hamiltonian, while now we use it equally in all terms of the Hamiltonian. The difference of these approximations directly corresponds to the difference of our goals in this and the preceeding section: there we aimed at a description of the Mott state, where the $\psi_{\sigma}$ both vanish, and its eventual instabilty toward the formation of a superfluid state with an incipient (small) nonvanishing $\psi_{\sigma}$, while here our goal is to describe the excitations deep in the superfluid states, where the mean field is large and contains practically all particles. The price we have to pay for achieving the latter goal is that the Mott state cannot be described by the modified form of the mean-field approximation.

For $\theta=0$ we can use Eqs. (15) to simplify the local meanfield Hamiltonian, which can then be written as

$$
H=-(\mu+2 J) n+\frac{U}{2} n^{2}-\frac{|P|}{2}\left(\alpha_{\Lambda}^{*} \alpha_{0} \pm \alpha_{0}^{*} \alpha_{\Lambda}\right)^{2},
$$

where "+" and "-" correspond to $g_{a}<0$ and $g_{a}>0$, respectively.

In the equlibrium state, we set the time derivatives of the mean fields to zero. Then it is easy to verify that the phases of $\alpha_{0}$ and $\alpha_{\Lambda}$ must be equal, and may be set to zero for convenience, i.e., we may, for the present purpose, simplify the mean-field ansatz

$$
\hat{a}_{\sigma i} \approx \sqrt{n_{\sigma}},
$$

$$
\begin{aligned}
i \hbar \frac{d}{d t} \hat{a}_{\Lambda i} & =-J_{\Lambda}\left(\hat{a}_{\Lambda, i-1}+\hat{a}_{\Lambda, i+1}\right)+U_{\Lambda} \hat{n}_{\Lambda i} \hat{a}_{\Lambda i} \\
& +K \hat{n}_{0 i} \hat{a}_{\Lambda i}-|P| \hat{a}_{\Lambda i}^{\dagger} \hat{a}_{0 i} \hat{a}_{0 i}-\mu \hat{a}_{\Lambda i} \\
i \hbar \frac{d}{d t} \hat{a}_{0 i} & =-J_{0}\left(\hat{a}_{0, i-1}+\hat{a}_{0, i+1}\right)+U_{0} \hat{n}_{0 i} \hat{a}_{0 i} \\
& +K \hat{n}_{\Lambda i} \hat{a}_{0 i}-|P| \hat{a}_{0 i}^{\dagger} \hat{a}_{\Lambda i} \hat{a}_{\Lambda i}-(\delta+\mu) \hat{a}_{0 i} .
\end{aligned}
$$

These equations remain valid in higher-dimensional lattices as
well with the only modification that the lattice indices aquire

In the mean-field approximation, the operators $\hat{a}_{\sigma i}$ are rethen finds a mean-field equation of the classical Hamiltonian

\section{s} . 
with the normalization condition $n_{\Lambda}+n_{0}=n$. It is the small but nonzero value of $|P|$ which aligns the phases of the $\hat{a}_{\sigma i}$ for $\sigma=0, \Lambda$. We are then left with the equations

$$
\begin{aligned}
& \mu \sqrt{n_{\Lambda}}=\left(-2 J_{\Lambda}+U_{\Lambda} n_{\Lambda}+K n_{0}-|P| n_{0}\right) \sqrt{n_{\Lambda}}, \\
& \mu \sqrt{n_{0}}=\left(-2 J_{0}+U_{0} n_{0}+K n_{\Lambda}-|P| n_{\Lambda}-\delta\right) \sqrt{n_{0}} .
\end{aligned}
$$

We consider the case $g_{a}>0$ first. Then eqs. 29) in general have no positive solutions for $n_{0}$ and $n_{\Lambda}$ with $n=n_{0}+n_{\Lambda}$. However, there is an exception for the special case $\theta=0$ where, as we have discussed, the usual U(1) symmetry of particle number conservation is enhanced to a $\mathrm{U}(1) \times \mathrm{U}(1)$ symmetry. As it was also mentioned above, it is in any case reasonable to consider only small values of $\theta$. Since the variation of $\delta$ with $\theta$ is much faster than that of $U_{0}, U_{\Lambda}$, and $P$, for the semiquantitative analysis one can put $U_{\Lambda} \approx U_{0}$ and $K \approx U_{0}+P$. Then Eqs. 29) take the form

$$
\begin{aligned}
& \mu \sqrt{n_{\Lambda}}=\left(-2 J_{\Lambda}+U_{0} n\right) \sqrt{n_{\Lambda}}, \\
& \mu \sqrt{n_{0}}=\left(-2 J_{0}+U_{0} n-\delta\right) \sqrt{n_{0}} .
\end{aligned}
$$

Let us assume that $n_{\Lambda} \neq 0, n_{0} \neq 0$. Then we can di- vide Eqs. 30 and 31 by $\sqrt{n_{0}}, \sqrt{n_{\Lambda}}$, respectively, and we see immediately that Eqs. (30), (31) are compartible only if $J_{\Lambda}=J_{0}+\delta / 2$, which does occur for $\theta=0$. Therefore in the degenerate case $\theta=0$ only the total density $n=n_{0}+n_{\Lambda}$ is determined,

$$
\mu=U n-2 J
$$

and the actual values taken by $n_{0}$ and $n_{\Lambda}$ break spontaneously the SU(2) symmetry by fixing an arbitrary orientation of $\mathbf{T}$ in the $(1,3)$ plane. For $\theta \neq 0$, either $n_{\Lambda}$ or $n_{0}$ must vanish, i.e., $\mathbf{T}$ is then oriented parallel or antiparallel to the 3 direction, respectively. Since the 0 mode has a lower energy, the solution of Eqs. (30) and (31) is

$$
n_{0}=n, \quad n_{\Lambda}=0, \quad \mu+\delta=U_{0} n-2 J_{0},
$$

exactly as in the case of one-component BEC [16, 17].

In the case $g_{a}<0$, the situation is different and the solution of Eq. 29) has the form

$$
\begin{aligned}
n_{\Lambda} & =\frac{\left(U_{0}-K+|P|\right) n-2\left(J_{0}-J_{\Lambda}\right)-\delta}{U_{\Lambda}+U_{0}-2(K-|P|)}, \quad n_{0}=\frac{\left(U_{\Lambda}-K+|P|\right) n+2\left(J_{0}-J_{\Lambda}\right)+\delta}{U_{\Lambda}+U_{0}-2(K-|P|)} \\
\mu & =-\left(J_{0}+J_{\Lambda}\right)+\frac{2\left(J_{0}-J_{\Lambda}\right)\left(U_{0}-U_{\Lambda}\right)-\delta\left(U_{\Lambda}+|P|-K\right)+\left[U_{0} U_{\Lambda}-(K-|P|)^{2}\right] n}{U_{\Lambda}+U_{0}-2(K-|P|)}
\end{aligned}
$$

provided that

$$
\left(U_{0}-K+|P|\right) n-2\left(J_{0}-J_{\Lambda}\right)-\delta>0,
$$

otherwise the solution is given by Eq. (33). For $n_{0}$ and $n_{\Lambda}$ both nonvanishing the Bose gas has a nonvanishing hyperfine and magnetization density in the $x$ direction proportional to $\sqrt{n_{0} n_{\Lambda}}$. form,

In the case $g_{a}<0, \theta=0$, Eqs. (34) take a very simple

$$
n_{0}=n_{\Lambda}=\frac{n}{2}, \quad \mu=(U-|P|) n-2 J .
$$

The excitations can be worked out employing the ansatz $\lfloor$, 17]

$$
\hat{a}_{\sigma i}=\sqrt{n_{\sigma}}+\hat{\Delta}_{\sigma i} .
$$

Substituting Eq. (37) into Eq. (24) we get the following lin- earized equations for the excitations:

$$
\begin{aligned}
& i \hbar \frac{d}{d t} \hat{\Delta}_{\Lambda i}=-J_{\Lambda}\left(\hat{\Delta}_{\Lambda, i-1}+\hat{\Delta}_{\Lambda, i+1}\right) \\
& +\left(2 U_{\Lambda} n_{\Lambda}+K n_{0}-\mu\right) \hat{\Delta}_{\Lambda i}+\left(U_{\Lambda} n_{\Lambda}-|P| n_{0}\right) \hat{\Delta}_{\Lambda i}^{\dagger} \\
& +(K-2|P|) \sqrt{n_{0} n_{\Lambda}} \hat{\Delta}_{0 i}+K \sqrt{n_{0} n_{\Lambda}} \hat{\Delta}_{0 i}^{\dagger}, \\
& i \hbar \frac{d}{d t} \hat{\Delta}_{0 i}=-J_{\Lambda}\left(\hat{\Delta}_{0, i-1}+\hat{\Delta}_{0, i+1}\right) \\
& +\left(2 U_{0} n_{0}+K n_{\Lambda}-\mu-\delta\right) \hat{\Delta}_{0 i}+\left(U_{0} n_{0}-|P| n_{\Lambda}\right) \hat{\Delta}_{0 i}^{\dagger} \\
& +(K-2|P|) \sqrt{n_{0} n_{\Lambda}} \hat{\Delta}_{\Lambda i}+K \sqrt{n_{0} n_{\Lambda}} \hat{\Delta}_{\Lambda i}^{\dagger} .
\end{aligned}
$$

From Eqs. 38 we obtain two coupled wave equations for the density fluctuations $\hat{\rho}_{\sigma i}=\sqrt{n_{\sigma}}\left(\hat{\Delta}_{\sigma i}+\hat{\Delta}_{\sigma i}^{\dagger}\right)$,

$$
\begin{aligned}
-\hbar^{2} \frac{d^{2}}{d t^{2}} \hat{\rho}_{\sigma i} & =J_{\sigma}^{2}\left(\hat{\rho}_{\sigma, i-2}+\hat{\rho}_{\sigma, i+2}\right) \\
& +A_{\sigma \sigma}^{(1)}\left(\hat{\rho}_{\sigma, i-1}+\hat{\rho}_{\sigma, i+1}\right)+A_{\sigma \sigma}^{(0)} \hat{\rho}_{\sigma i} \\
& +A_{\sigma \sigma^{\prime}}^{(1)}\left(\hat{\rho}_{\sigma^{\prime}, i-1}+\hat{\rho}_{\sigma^{\prime}, i+1}\right)+A_{\sigma \sigma^{\prime}}^{(0)} \hat{\rho}_{\sigma^{\prime} i} .
\end{aligned}
$$

The coefficients $A_{\sigma \sigma^{\prime}}^{(0,1)}$ are listed in the Appendix. The eigenmodes can be found by means of Fourier transformation,

$$
\hat{\rho}_{\sigma i}=\sum_{k} c_{\sigma k} \hat{b}_{k} \exp \left[i\left(k z_{i}-\omega_{k} t\right)\right] .
$$


In the case $\theta=0$, the two wave equations for the sum and the difference of the two densities are decoupled. The coefficients $c_{\sigma k}$ are then independent of $k$. For $g_{a}<0$ the two eigenmodes are given by

$$
\begin{aligned}
& \hbar \omega_{k}^{(+)}=\sqrt{\mathcal{E}(k)[\mathcal{E}(k)+2(U-|P|) n]}, c_{\Lambda}^{(+)} / c_{0}^{(+)}=1, \\
& \hbar \omega_{k}^{(-)}=\mathcal{E}(k)+2|P| n, \quad c_{\Lambda}^{(-)} / c_{0}^{(-)}=-1,
\end{aligned}
$$

and for $g_{a}>0$ by

$$
\begin{array}{ll}
\hbar \omega_{k}^{(+)}=\sqrt{\mathcal{E}(k)[\mathcal{E}(k)+2 U n]}, & c_{\Lambda}^{(+)} / c_{0}^{(+)}=1,(42) \\
\hbar \omega_{k}^{(-)}=\sqrt{\mathcal{E}(k)[\mathcal{E}(k)+2 P n]}, & c_{\Lambda}^{(-)} / c_{0}^{(-)}=-1,
\end{array}
$$

where

$$
\mathcal{E}(k)=4 J \sin ^{2}\left(\frac{\pi}{2} \frac{k}{k_{L}}\right)
$$

is a dispersion relation of free particles in a lattice. The symmetric modes with the eigenfrequencies $\omega_{k}^{(+)}$are qualitatively the same in both cases $\left(g_{a}<0\right.$ and $\left.g_{a}>0\right)$. They are the Goldstone modes of the spontaneous breaking of the $\mathrm{U}(1)$ symmetry of particle number conservation, which correspond to condensate excitations. The antisymmetric mode is principally different: In the case $g_{a}>0$, it is an additional Goldstone mode [in which the components $\delta T_{\perp}$ transverse to the isospin vector in the $(1,3)$ plane oscillate] corresponding to the spontaneous breaking of the isospin symmetry, by the ground state; but for $g_{a}<0$ the isospin is oriented along the 1-direction by the interaction and the isospin symmetry around this axis is not spontaneously broken. In the antisymmetric mode there is therefore a gap of size $2|P| n$, and $\left(\omega_{k}^{(-)}-2|P| n\right) \sim k^{2}$ for small $k$. This is again an isospin wave with $\delta T_{2}$ and $\delta T_{3}$ propagating together. In the case $g_{a}<0$, the gap allows the energies of the symmetric and antisymmetric modes to cross at the points

$$
\frac{k}{k_{L}}= \pm \arcsin \frac{2}{\pi} \sqrt{\frac{|P|^{2} n}{2 J(U-3|P|)}} .
$$

In the case $\theta \neq 0$, there are also two excitation modes. Let us consider the case $g_{a}<0$ first. Typical $k$ dependences for $n_{\Lambda} \neq 0, n_{0} \neq 0$, are shown in Fig. 10 The two branches do not cross anymore and the coefficients $c_{\Lambda k}, c_{0 k}$ depend strongly on $k$ near the points of avoided crossings (44), where the type of oscillations changes. If we increase $\theta$ higher then a certain value the condition (35) is not fulfilled. According to Eq. (33), Eqs. (38) become decoupled and the energies of the eigenmodes are given by

$$
\begin{aligned}
\hbar \omega_{0 k}= & \sqrt{\mathcal{E}_{0}(k)\left(\mathcal{E}_{0}(k)+2 U_{0} n\right)} \\
\hbar \omega_{\Lambda k}= & \left\{\left[\mathcal{E}_{\Lambda}(k)-\left(U_{0}-K\right) n+2\left(J_{0}-J_{\Lambda}\right)+\delta\right]^{2}\right. \\
& \left.-(P n)^{2}\right\}^{1 / 2}
\end{aligned}
$$

where $\mathcal{E}_{0(\Lambda)}$ is given by Eq. (43) with $J$ replaced by $J_{0(\Lambda)}$. Since inequality (35) does not hold and $\mathcal{E}_{\Lambda}(0)=0$, the $\Lambda$
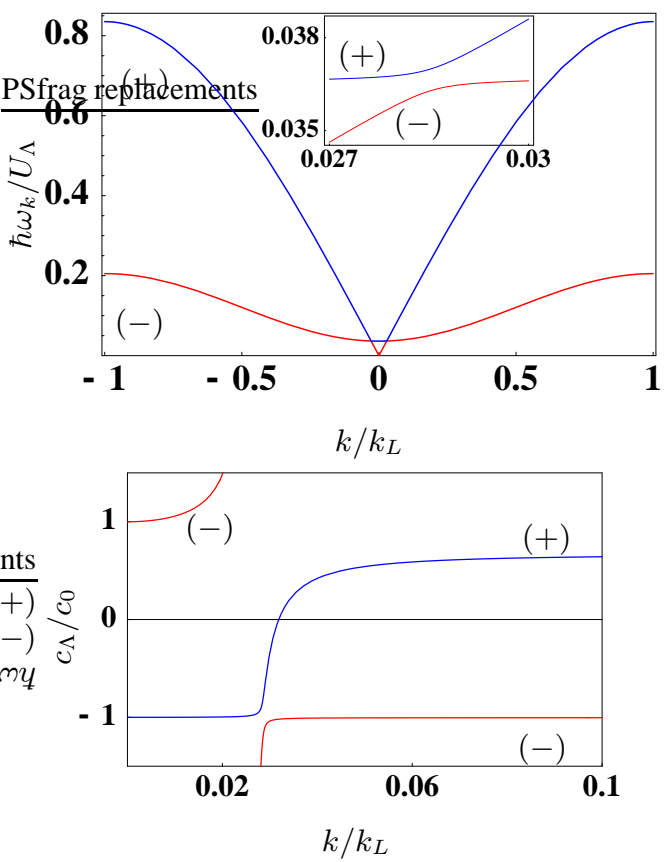

FIG. 10: Spectrum of collective excitations $\hbar \omega_{k}$ and the ratio $c_{\Lambda k} / c_{0 k}$ for ${ }^{87} \mathrm{Rb}\left(g_{a}<0\right) . q=\tilde{\Omega}_{0}^{2} / 4 \omega_{R} \Delta=-4.5, \theta=0.7^{\circ}$, $n=2$.

mode has a gap at $k=0$. Moreover, $U_{0}-K(\approx|P|)$ is positive as well as all other summands in the square brackets in Eq. (46). This means that for some nonvanishing values of $k$ and large enough $n$ the frequency $\omega_{\Lambda k}$ can become imaginary, i.e., the system can be unstable.

In the case $g_{a}>0, \theta \neq 0$, the excitation modes are given by Eqs. (45) and (46), and $K-U_{0}(\approx P)$ is positive. Therefore the frequencies $\omega_{\Lambda k}$ are always positive and there is a gap at $k=0$. For $\theta=0$ this spectrum reduces to that given by Eq. (42) as it should.

\section{CONCLUSIONS}

We have investigated QPT of spin-1 bosons in an optical lattice created by the lin- $\theta$-lin laser configuration. The laser beams create not only periodic potentials for atoms, but also couple the atomic ground states with magnetic quantum numbers $m= \pm 1$. As a result there are only two lowest-energy Bloch bands $(0$ and $\Lambda$ ) which should be taken into account and the original three-component system is reduced to a twocomponent one.

The interaction between the atoms is characterized by two scattering lengths, the symmetric $g_{s}$ and the much smaller but very important asymmetric $g_{a}$. This setup could be realized with $\mathrm{Rb}$ or $\mathrm{Na}$ atoms, for which $g_{a}$ is negative (ferromagnetic interaction) and positive (antiferromagnetic interaction), respectively. It turned out that an appropriate change of the easily tunable parameters of the setup, the laser intensity, and the angle $\theta$, permits a very rich scenario of phase transitions which we explored in detail in Section IV. The symmetries of the Hamiltonian are discussed and it is shown that the proper- 
ties are essentially different for the atoms with ferromagnetic and antiferromagnetic interactions. Depending on the sign of the asymmetric scattering length, the isospin symmetry is or is not spontaneously broken in the superfluid phase. The corresponding collective modes, besides the always present Bogoliubov mode, are isospin waves and either gapless or gapped at $k=0$. A surprising feature we found in this study is that the phase transition between Mott and superfluid phase can also be of first order in certain domains of parameter space, with associated domains where both phases can coexist, but, as we found, this is only possible for $g_{a}>0$.

\section{Acknowledgments}

This work has been supported by the SFB/TR 12 "Symmetries and universalities in mesoscopic physics".

\section{APPENDIX: COEFFICIENTS IN EQ. (39)}

The coefficients in Eq. (39) are given by

$$
\begin{aligned}
A_{\Lambda \Lambda}^{(1)}= & -2 J_{\Lambda}\left(2 U_{\Lambda} n_{\Lambda}+K n_{0}-\mu\right) \\
A_{\Lambda 0}^{(1)}= & 2\left[J_{0}|P|-J_{\Lambda}(K-|P|)\right] n_{\Lambda} \\
A_{\Lambda \Lambda}^{(0)}= & 2 J_{\Lambda}^{2}-4|P|(K-|P|) n_{0} n_{\Lambda} \\
& +\left[U_{\Lambda} n_{\Lambda}+(K+|P|) n_{0}-\mu\right] \\
& \times\left[3 U_{\Lambda} n_{\Lambda}+(K-|P|) n_{0}-\mu\right] \\
A_{\Lambda 0}^{(0)}= & 2 n_{\Lambda}\left\{\left[U_{\Lambda} n_{\Lambda}+(K+|P|) n_{0}-\mu\right](K-|P|)\right. \\
& \left.-|P|\left[3 U_{0} n_{0}+(K-|P|) n_{\Lambda}-(\mu+\delta)\right]\right\}
\end{aligned}
$$

Expressions for $A_{00}^{(1)}, A_{0 \Lambda}^{(1)}, A_{00}^{(0)}$, and $A_{0 \Lambda}^{(0)}$ can be obtained from these equations making the replacement $\Lambda \rightarrow 0,0 \rightarrow \Lambda$, $\mu \rightarrow(\mu+\delta),(\mu+\delta) \rightarrow \mu$.
[1] M. Greiner et al., Nature (London) 415, 39 (2002).

[2] E. Demler and F. Zhou, Phys. Rev. Lett. 88, 163001 (2002).

[3] S. K. Yip, Phys. Rev. Lett. 90, 250402 (2003).

[4] F. Zhou, Europhys. Lett. 63, 505 (2003).

[5] A. Imambekov, M. Lukin, and E. Demler, Phys. Rev. A 68, 063602 (2003).

[6] M. Snoek and F. Zhou, Phys. Rev. B 69, 094410 (2004).

[7] A. A. Svidzinsky and S. T. Chui, Phys. Rev. A 68, 043612 (2003).

[8] J.-M. Hou and M.-L. Ge, Phys. Rev. A 67, 063607 (2003).

[9] K. V. Krutitsky and R. Graham, Phys. Rev. Lett. 91, 240406 (2003).
[10] G. Grynberg and C. Robilliard, Phys. Rep. 355, 335 (2001).

[11] T.-L. Ho, Phys. Rev. Lett. 81, 742 (1998).

[12] T. Ohmi and K. Machida, J. Phys. Soc. Jpn. 67, 1822 (1998).

[13] V. Dunjko et al., Phys. Rev. Lett. 86, 5413 (2001).

[14] D. Jaksch et al., Phys. Rev. Lett. 81, 3108 (1998).

[15] S. Sachdev, Quantum phase transitions (Cambridge University Press, Cambridge, England, 2001).

[16] D. van Oosten, P. van der Straten, and H. T. C. Stoof, Phys. Rev. A 63, 053601 (2001).

[17] A. M. Rey et al., J. Phys. B 36, 825 (2003). 